\title{
A randomized, double-blind, placebo-controlled study of the effect of ezetimibe on glucose metabolism in subjects with type 2 diabetes mellitus and hypercholesterolemia
}

\author{
Itori Saito', Kyoichi Azuma', Taro Kakikawa" ${ }^{1 *}$, Nobuyuki Oshima', Mary E Hanson² and Andrew M Tershakovec ${ }^{2}$
}

\begin{abstract}
Background: Recent evidence points to an increased incidence of new-onset diabetes and a negative impact on glucose parameters with statin use. This study examined the safety of ezetimibe vs placebo for change from baseline to week 24 in HbA1c (primary endpoint), glycoalbumin, and fasting plasma glucose (secondary endpoints) in Japanese subjects with type 2 diabetes and hypercholesterolemia.
\end{abstract}

Methods: This was a randomized, double-blind, placebo-controlled, parallel-group, multi-site trial. Adults with type 2 diabetes and hypercholesterolemia whose LDL-C measured $<140 \mathrm{mg} / \mathrm{dl}$ (subjects receiving lipid-lowering drugs) or $<160 \mathrm{mg} / \mathrm{dl}$ (subjects not receiving lipid-lowering drugs) at the start of the screening phase, were randomized after a 5-week wash-out period to ezetimibe $10 \mathrm{mg}$ or placebo (1:1) for 24 weeks. Changes in HbA1c, glycoalbumin and fasting plasma glucose from baseline to week 24 were evaluated. The non-inferiority margin was set at $0.5 \%$ for HbA1c.

Results: Overall, 152 subjects were randomized (75 to ezetimibe and 77 to placebo). From baseline to 24 weeks, HbA1c significantly increased in both the ezetimibe and placebo groups (between-treatment difference 0.08 [95\% Cl: -0.07 to 0.23]). Ezetimibe was statistically non-inferior to placebo. At 24 weeks, the mean change from baseline in glycoalbumin levels (between-treatment differences 0.00 [95\% Cl: -0.47, 0.47]) and fasting plasma glucose (between-treatment differences $-4.8[95 \% \mathrm{Cl}:-12.1,2.1])$ were similar in both treatment groups.

Conclusions: These results suggest that ezetimibe $10 \mathrm{mg}$ does not result in dysregulation of glucose metabolism in Japanese patients with type 2 diabetes and hypercholesterolemia over 24 weeks of treatment.

Trial registration: ClinicalTrials.gov identifier NCT01611883.

Keywords: Fasting plasma glucose, Glycoalbumin, HbA1c, Japanese, LDL-C

\section{Background}

While diabetes, hypertension and hypercholesterolemia are each independent risk factors for coronary artery disease (CAD), concomitant occurrence of these factors may lead to a cumulative increase in CAD risk [1]. Management of these risk factors is important for reducing the risk of atherosclerosis and the risk of ischemic heart disease and CAD. In addition to lipid management and blood pressure control, glycemic control is a basic component in

\footnotetext{
* Correspondence: taro.kakikawa@merck.com

'MSD KK, Japan development, Clinical research, Kitanomaru Square, 1-13-12 Kudan-kita, Chiyoda-ku 102-8667, Tokyo, Japan

Full list of author information is available at the end of the article
}

the management of diabetes and is achieved in part via diabetes self-management, education, exercise and improved diet, the latter elements being the cornerstones of treatment for diabetes and lipids. As such, it is important that glucose metabolism not be adversely affected when patients take lipid-lowering treatment.

Post-hoc analyses of large clinical trials and metaanalyses have demonstrated a dose- and potencydependent effect for increased incidence of new-onset diabetes and mild elevation of hemoglobin A1c (HbA1c) and fasting plasma glucose with statin use [2-7]. However, it was determined that the benefits of reducing the risk of cardiovascular events with statin use significantly outweigh the 
potential risks of worsening glycemic control or developing new-onset diabetes [8]. Ezetimibe is an inhibitor of a small intestine cholesterol transporter. It lowers low-density lipoprotein cholesterol (LDL-C) by 15-20\% through inhibition of exogenous and biliary cholesterol absorption in the digestive tract by inhibiting the Niemann-Pick C1-Like 1 in the brush border of enterocytes $[9,10]$. A significant reduction in insulin resistance and/or fatty liver have been reported in small clinical trials using the combination of pravastatin with ezetimibe [11] or ezetimibe monotherapy $[12,13]$. However, other small studies have observed no changes in parameters of glucose metabolism and insulin sensitivity with ezetimibe monotherapy [14-16]. Limited data suggest that ezetimibe, whether added to a statin or used as a monotherapy, does not have an adverse effect on glucose metabolism [17,18]. However, in light of recent findings regarding the increased incidence of new-onset diabetes and the impact on glucose parameters with the use of statins [2-7], it is important to assess any potential for these same issues with ezetimibe use. This study was conducted to prospectively examine the effects of ezetimibe on glucose metabolism in patients with type 2 diabetes and hypercholesterolemia.

The primary objective of this study was to examine the safety of ezetimibe compared with placebo with regard to change in HbA1c from baseline to week 24 in subjects with type 2 diabetes and hypercholesterolemia. Secondary objectives included comparing ezetimibe to placebo in subjects with type 2 diabetes and hypercholesterolemia for change in glycoalbumin and fasting plasma glucose from baseline to week 24; the proportion of patients having onset of "exacerbation of diabetes mellitus" (assessed by index of blood glucose control, changes in diabetes medications, and compliance to diet and exercise therapy); the proportion of patients with changes to diabetes medications due to worsening of diabetes.

\section{Methods Study design}

This was a randomized, double-blind, placebo-controlled, parallel-group, multi-site trial (protocol number: PN367, Additional file 1; ClinicalTrials.gov identifier NCT01611883) of ezetimibe in subjects with type 2 diabetes and hypercholesterolemia carried out at 19 sites in Japan and conducted in conformance with Good Clinical Practice and the Declaration of Helsinki between August 2012 and January 2014. The protocol was approved by the institutional review boards for each study site (Japanese Association for the Promotion of State of the Art in Medicine - The Second Institutional Review Board; Tokushukai Group Institutional Review Board; Hirokuni Hospital Institutional Review Board; Doujin Memorial Foundation Meiwa Hospital Institutional Review Board; Clinical Research Promotion Network Japan Institutional
Review Board; Hatamoto Institutional Review Board). All patients provided written, informed consent prior to entering the study. Each subject participated in the trial for approximately 33 weeks from the time the subject signed the informed consent form through the final contact. After screening, eligible subjects stopped any current lipidlowering medications for 5 weeks prior to receiving assigned treatment for approximately 24 weeks. Ezetimibe $10 \mathrm{mg}$ or placebo was administered orally once daily and follow-up visits occurred at 4, 12, 20 and 24 weeks of treatment. Serious adverse events were monitored from the time informed consent was obtained until 30 days after the administration of the last dose of study drug.

\section{Subjects}

Subjects with a diagnosis of type 2 diabetes mellitus and hypercholesterolemia aged 20 to 75 years were selected to participate in the trial, including those undergoing treatment with oral anti-diabetic drugs or insulin or both, and who had no change in the type, dose, and regimen of drugs for the treatment of diabetes within 12 weeks before the start of the screening phase. However, small changes in insulin dosing $+/-5 \mathrm{U}$ were acceptable. Subjects undergoing diet and exercise therapy with no change in either therapy within 4 weeks before the start of the screening phase (however, exercise therapy was not necessarily applicable in the case of patients with coexisting conditions judged not appropriate to meet this criterion). Subjects were included whose LDL-C measured at the start of the screening phase < $140 \mathrm{mg} / \mathrm{dl}$ if they had been receiving lipid lowering drugs; in subjects who had not been receiving lipid lowering drugs, LDL-C measures $<160 \mathrm{mg} / \mathrm{dl}$ were required. At 1 week prior to randomization, all subjects were required to have LDL-C level $\geq 120 \mathrm{mg} / \mathrm{dl}$ and $<160 \mathrm{mg} / \mathrm{dl}$ in order to participate in the study. Subjects were excluded if their triglyceride value exceeded $400 \mathrm{mg} / \mathrm{dl}$, HbA1c value was $\geq 8.4 \%$, (HbA1c was recorded in the National Glycohemoglobin Standardization Program [NGSP]), and/ or fasting plasma glucose was $\geq 170 \mathrm{mg} / \mathrm{dl}$ at screening or 1 week prior to randomization. Subjects were excluded if they had history of coronary heart disease (CHD), stroke, or arteriosclerosis obliterans; if they had hypercholesterolemia associated with hypothyroidism; or if they had active or severe hepatic disease. Concomitant therapy with other lipid modifying therapies including statins, fibrates, niacin, cyclosporine, systemic corticosteroids, and investigational drugs was prohibited.

\section{Safety and efficacy endpoints}

The primary endpoint of this study was to examine the safety of ezetimibe compared with placebo with regard to change in HbA1c from baseline to week 24. Secondary endpoints included comparing ezetimibe to placebo 
for change in glycoalbumin and fasting plasma glucose from baseline to week 24. Additionally, the proportion of subjects having exacerbation of diabetes mellitus (exacerbation of diabetes mellitus was judged by the investigator taking into account the blood glucose control indices, diabetes medications, and compliance to diet and exercise therapy) and the proportion of subjects with changes in diabetes medication due to worsening of diabetes was calculated (small changes in insulin dosing $\pm 5 \mathrm{U}$ were excluded). Additional safety was monitored through collection of adverse events (AEs), adverse drug reactions, hematology (white blood cell count, red blood cell count, $\mathrm{Hb}$, hematocrit, platelet count), biochemistry (aspartate aminotransferase, alanine aminotransferase, gamma glutamyl transpeptidase, alkaline phosphatase, lactate dehydrogenase, creatine phosphokinase, total bilirubin, direct bilirubin, total protein, blood urea nitrogen, uric acid, creatinine, $\mathrm{Na}, \mathrm{K}, \mathrm{Cl}$ ) and vital signs (body weight, blood pressure, pulse) throughout the study. For efficacy endpoints the percent change from baseline to week 24 in serum lipids, including LDL-C, estimated by the Friedewald equation, total cholesterol, triglycerides, and high-density lipoprotein cholesterol (HDL-C) were measured. Non-HDL-C was calculated by subtracting HDL-C from total cholesterol. All laboratory measurements, including glucose metabolism and lipid parameters, were conducted at a central laboratory.

\section{Statistical analysis}

One-hundred-forty eight subjects were planned for enrollment with at least $45(30 \%)$ receiving insulin. For the primary safety hypothesis of this study, the difference between the ezetimibe and placebo groups in the least squares mean change in $\mathrm{HbA1c}$ from baseline to 24 weeks after treatment was assumed to be $0.1 \%$, with standard deviation of $0.7 \%$ and a non-inferiority margin of $0.5 \%$. The standard deviation and between groups difference were determined using a previous open-label study (a Phase III, trial in diabetic mellitus subjects with hypercholesterolemia) which provided a limited number of patients. The non-inferiority margin was given as a clinically meaningful exacerbation of diabetes control in medical practice. With this hypothesis, the number of subjects per group was projected to be 66 with a twosided 5\% (one-sided 2.5\%) probability of type 1 error, $90 \%$ power, and 1:1 allocation to each of the groups. The sample size was set to 148 to take into account a $10 \%$ dropout rate. There is only one primary hypothesis for this study; therefore, the issue of multiplicity will not occur.

The data set for the statistical analysis of safety is the per protocol set (PPS). Missing values were not replaced with other values. The full analysis set (FAS) was used for the sensitivity analysis of the primary trial objective and was defined as all randomized subjects meeting the inclusion criteria who received study drug and for whom a baseline and at least one post-baseline measurement was obtained. This was the data set for the efficacy analysis. Missing values were not replaced with other values. The PPS was defined as all randomized subjects meeting inclusion criteria who were not excluded from the FAS and were at least $75 \%$ compliant with study medication. The all subjects as treated (ASaT) group was defined as all subjects who took at least one dose of study drug during the treatment period. The ASaT was used for the analysis of AEs.

The longitudinal analysis of covariance was used to assess the primary (HbA1c) and secondary safety endpoints (glycoalbumin and fasting plasma glucose). Baseline, treatment group, HbA1c ( 2 categories: $<7.4 \%$ and $\geq 7.4 \%$ to $<8.4 \%$ ), insulin use ( 2 categories: yes, no), time, and "time $\times$ treatment group" interaction were included in the model. So as not to place restriction on the time-course curve of mean values, time was a categorical variable. Between-group difference in mean amount of change from baseline at measurement time points was estimated and tested. Correlation between time-course measured values was modeled using an unstructured covariance matrix, but if this calculation did not converge, Toeplitz was used for correlation. Based on the above unstructured model, the difference in least squares (LS) mean from the placebo group and the 95\% confidence interval $(\mathrm{CI})$ were calculated. The LS mean value of the amount of change from baseline with each group and the $95 \%$ CI were also calculated. As sensitivity analysis of the primary endpoint, the same analysis was conducted with the FAS. A between-group comparison was conducted using Fisher's exact test for the proportion of subjects with "exacerbation of diabetes". A between-group comparison using Fisher's exact test was also conducted for the proportion of subjects with changes in diabetes medications due to worsening of diabetes. Summary statistics with standard deviations were calculated for demographic and baseline characteristics and the number of subjects with AEs and the incidence of AEs were summarized by treatment group and by system organ class and event. The percent change from baseline to each measurement time point was calculated by treatment group for lipid parameters using the same longitudinal analysis of covariance used for the primary safety endpoint. Lipids included LDL-C, total cholesterol, triglycerides, HDL-C, and non-HDL-C. No adjustments were made for multiplicity.

\section{Results}

A total of 342 subjects were screened, resulting in 152 randomized to treatment ( 75 to ezetimibe $10 \mathrm{mg}$ and 77 to placebo; Figure 1). In each treatment group, only 1 subject discontinued due to an AE. No patients were 


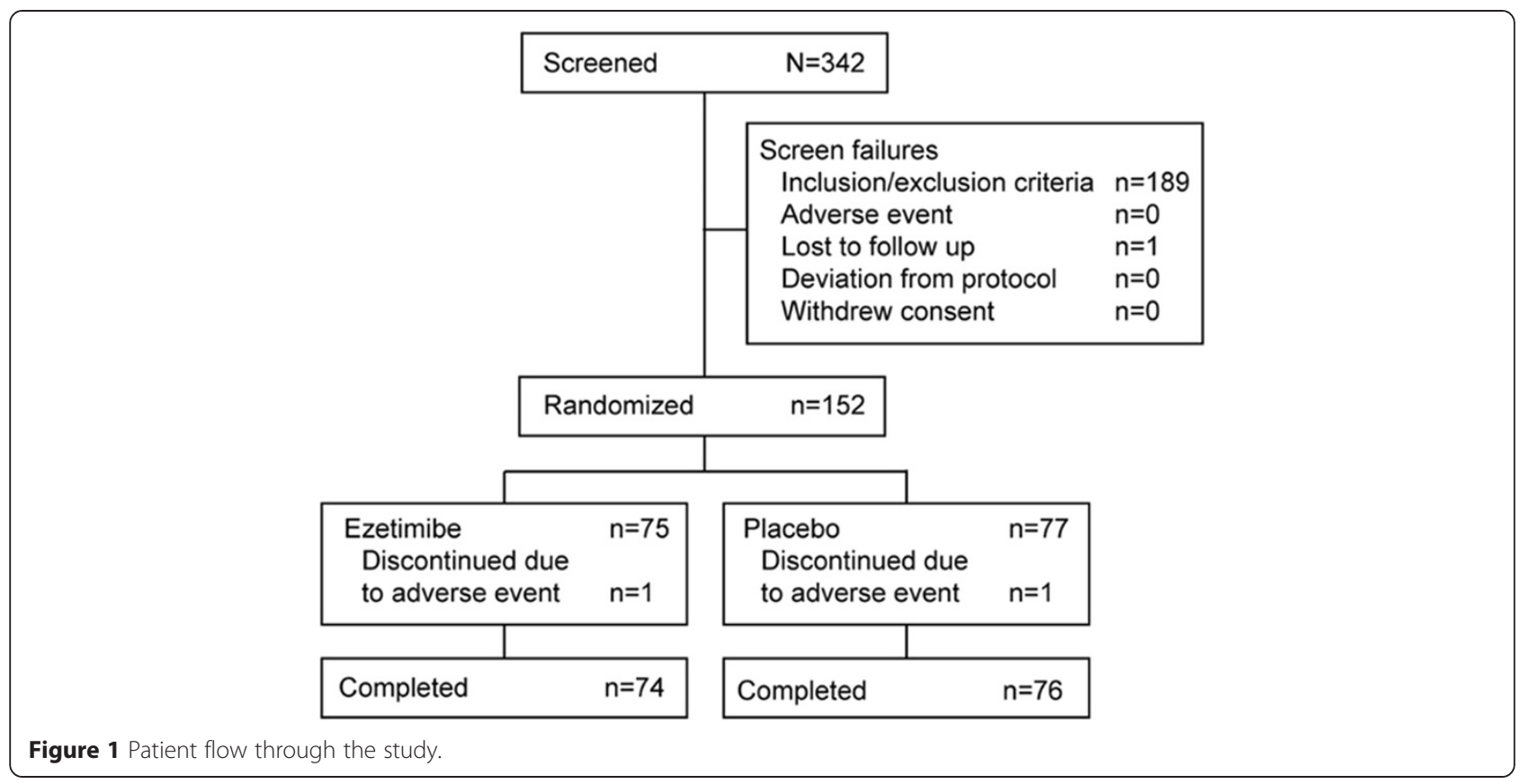

excluded from the safety and efficacy analyses. Baseline demographics and clinical characteristics were similar between treatment groups (Table 1). Approximately $2 / 3$ of subjects were male, the mean ( \pm standard deviation [SD]) age was $60( \pm 10)$ years, with a mean $( \pm \mathrm{SD})$ body mass index of $26( \pm 5) \mathrm{kg} / \mathrm{m}^{2}$. The vast majority (95\%) of subjects had coexisting diseases. There was no notable difference observed between the two groups in coexisting diseases or the use of concomitant medications (Table 1). At baseline mean HbA1c was $7.0 \%$, glycoalbumin was $17.3 \%$, and fasting plasma glucose was $126.3 \mathrm{mg} / \mathrm{dL}$.

The mean baseline values and change from baseline in blood glucose control indices in the ezetimibe $10 \mathrm{mg}$ and placebo groups are shown in Table 2. Over the course of the 24 weeks of treatment, HbA1c significantly increased from baseline in both the ezetimibe $10 \mathrm{mg}$ and placebo groups (Figure 2). At 24 weeks, the least-squares (LS) mean (95\% CIs) changes from baseline in HbA1c were 0.22 (0.11 to 0.34$)$ and 0.14 (0.03 to 0.25$)$ for the ezetimibe $10 \mathrm{mg}$ and placebo groups, respectively (Table 2 ). The between-treatment difference in the change from baseline was 0.08 ( -0.07 to 0.23$)$. The ezetimibe $10 \mathrm{mg}$ group was statistically non-inferior to the placebo group, compared with the pre-defined noninferiority margin of 0.5 (Table 2). The sensitivity analysis was consistent with the primary results, demonstrating non-inferiority of ezetimibe. Results were generally consistent across subgroups defined by baseline $\mathrm{HbA1c}$ level ( $<7.4 \%$, vs. $\geq 7.4 \%$ to $<8.4 \%)$ and by insulin administration at baseline (yes vs. no; data not shown).

At 24 weeks, the LS mean changes (95\% CIs) from baseline in glycoalbumin were $-0.02(-0.37,0.34)$ and -0.02
$(-0.37,0.33)$ for the ezetimibe $10 \mathrm{mg}$ and placebo groups, respectively (Table 2). Over the course of the 24 weeks of treatment, changes from baseline in glycoalbumin levels in both treatment groups were similar (Figure 3). The between-treatment difference in the change from baseline at 24 weeks was $(0.00 ; 95 \% \mathrm{CI}:-0.47,0.47$; Table 2$)$.

At 24 weeks, the LS mean changes (95\% CIs) from baseline in fasting plasma glucose were $6.6(1.1,12.1)$ and 11.4 $(6.1,16.7)$ for the ezetimibe $10 \mathrm{mg}$ and placebo groups, respectively (Table 2). Over the course of the 24 weeks of treatment, fasting plasma glucose significantly increased from baseline in both treatment groups (Figure 4). The between-treatment difference in the change from baseline at 24 weeks was $(-4.8 ; 95 \%$ CI: $-12.1,2.1$; Table 2$)$.

There was no significant difference in exacerbation of diabetes between treatment groups $(p=0.78)$. Seven subjects $(9 \%)$ in the ezetimibe group and 6 subjects $(8 \%)$ in the placebo group experienced exacerbation of diabetes. Similarly, there was no significant difference between groups in the proportion of subjects with changes to diabetes medications due to worsening of diabetes $(p=0.37)$. Seven subjects (9\%) in the ezetimibe group and 4 subjects $(5 \%)$ in the placebo group made changes to their diabetes medications due to worsening of their diabetes during the study period.

The percent changes from baseline to 24 weeks in LDL-C, total cholesterol, triglycerides, HDL-C and nonHDL-C are shown in Figure 4. After 24 weeks of treatment, the change from baseline was statistically significant for all 5 lipid parameters in subjects treated with ezetimibe $10 \mathrm{mg}(\mathrm{P}<0.05)$ but was not statistically significant in subjects who received placebo. The percent differences $(95 \%$ 
Table 1 Baseline demographics and clinical characteristics

\begin{tabular}{|c|c|c|c|}
\hline & Ezetimibe & Placebo & Total \\
\hline Subjects in population & 75 & 77 & 152 \\
\hline Male, n (\%) & $46(61.3)$ & $48(62.3)$ & $94(61.8)$ \\
\hline Mean age, yrs (SD) & $59.3(10.8)$ & $60.0(9.7)$ & $59.6(10.2)$ \\
\hline Mean body mass index, kg/m2 (SD) & $26.2(5.3)$ & $25.7(3.9)$ & $25.9(4.6)$ \\
\hline Coexisting diseases, yes; n (\%) & $70(93.3)$ & $74(96.1)$ & $144(94.7)$ \\
\hline \multicolumn{4}{|l|}{ Selected coexisting diseases, n (\%): } \\
\hline Cardiac disease other than CHD & $3(4.0)$ & $4(5.2)$ & $7(4.6)$ \\
\hline Hypertension & $37(49.3)$ & $35(45.5)$ & $72(47.4)$ \\
\hline Hepatic steatosis & $24(32.0)$ & $26(33.8)$ & $50(32.9)$ \\
\hline Hyperuricaemia & $6(8.0)$ & $7(9.1)$ & $13(8.6)$ \\
\hline Obesity & $2(2.7)$ & $1(1.3)$ & $3(2.0)$ \\
\hline Concomitant medications, yes; $\mathrm{n}(\%)$ & $75(100)$ & $77(100)$ & $152(100)$ \\
\hline \multicolumn{4}{|l|}{ Selected concomitant medications, n (\%): } \\
\hline Oral anti diabetes agents & $70(93.3)$ & $72(93.5)$ & $142(93.4)$ \\
\hline Dipeptidyl peptidase-4 inhibitors & $39(52.0)$ & $29(37.7)$ & $68(44.7)$ \\
\hline Sulfonylureas & $27(36.0)$ & $22(28.6)$ & $49(32.2)$ \\
\hline Glinides & $3(4.0)$ & $10(13.0)$ & $13(8.6)$ \\
\hline Thiazolidine & $12(16.0)$ & $9(11.7)$ & $21(13.8)$ \\
\hline Biguanides & $34(45.3)$ & $28(36.4)$ & $62(40.8)$ \\
\hline Alpha-glucosidase inhibitors & $24(32.0)$ & $24(31.2)$ & $48(31.6)$ \\
\hline Thiazolidine + Biguanides & $1(1.3)$ & - & $1(0.7)$ \\
\hline Glinides + Alpha-glucosidase inhibitors & $2(2.7)$ & - & $2(1.3)$ \\
\hline Insulin administration yes; $\mathrm{n}(\%)$ & $23(30.7)$ & $24(31.2)$ & $47(30.9)$ \\
\hline Hypertension agents & $35(46.7)$ & $30(39.0)$ & $65(42.8)$ \\
\hline Calcium channel blocker & $19(25.3)$ & $15(19.5)$ & $34(22.4)$ \\
\hline Beta blocker & $2(2.7)$ & $1(1.3)$ & $3(2.0)$ \\
\hline Alpha blocker & $5(6.7)$ & $2(2.6)$ & $7(4.6)$ \\
\hline ARB & $22(29.3)$ & $17(22.1)$ & $39(25.7)$ \\
\hline Diuretic & $3(4.0)$ & $1(1.3)$ & $4(2.6)$ \\
\hline ACE inhibitor & $2(2.7)$ & $4(5.2)$ & $6(3.9)$ \\
\hline ARB + Diuretic & $3(4.0)$ & $1(1.3)$ & $4(2.6)$ \\
\hline ARB + Calcium channel blocker & $3(4.0)$ & $3(3.9)$ & $6(3.9)$ \\
\hline Mean duration of diabetes, months (SD) & $88.9(74.0)$ & $94.9(93.8)$ & $92.0(84.4)$ \\
\hline Mean $\mathrm{HbA1c}$ \% (SD) & $7.0(0.6)$ & $7.0(0.6)$ & $7.0(0.6)$ \\
\hline$<7.4 \%, \mathrm{n}(\%)$ & $56(74.7)$ & $58(75.3)$ & $114(75.0)$ \\
\hline$\geq 7.4 \%,<8.4 \%, n(\%)$ & $19(25.3)$ & $19(24.7)$ & $38(25.0)$ \\
\hline Mean glycoalbumin, \% (SD) & $17.3(2.3)$ & $17.4(2.6)$ & $17.3(2.4)$ \\
\hline Mean fasting plasma glucose, mg/dL (SD) & $125.7(22.2)$ & $126.9(17.3)$ & $126.3(19.8)$ \\
\hline Mean LDL-C, mg/dL (SD) & $138.6(11.2)$ & $139.4(10.4)$ & $139.0(10.8)$ \\
\hline Mean total cholesterol, mg/dL (SD) & $217.6(16.2)$ & $219.0(21.1)$ & $218.3(18.8)$ \\
\hline Mean triglycerides, mg/dL (SD) & $119.6(57.3)$ & $129.5(63.8)$ & $124.6(60.7)$ \\
\hline Mean HDL-C, mg/dL (SD) & $55.0(13.5)$ & $53.7(12.8)$ & $54.3(13.1)$ \\
\hline Mean non-HDL-C, mg/dL (SD) & 162.6 (15.2) & $165.3(16.4)$ & $164.0(15.9)$ \\
\hline
\end{tabular}

$\mathrm{ARB}=$ angiotensin receptor blocker; $\mathrm{ACE}=$ angiotensin converting enzyme; $\mathrm{HbA1c}=$ hemoglobin $\mathrm{A} 1 \mathrm{c} \mathrm{HDL-C}=$ high-density lipoprotein cholesterol; LDL-C = lowdensity lipoprotein cholesterol. 
Table $\mathbf{2}$ Change from baseline to $\mathbf{2 4}$ weeks in glucose parameters

\begin{tabular}{|c|c|c|c|c|c|c|}
\hline & \multirow[b]{2}{*}{ Treatment (n) } & \multirow{2}{*}{$\begin{array}{l}\text { Baseline } \\
\text { Mean (SE) }\end{array}$} & \multirow{2}{*}{$\begin{array}{l}\text { Week } 24 \\
\text { Mean (SE) }\end{array}$} & \multicolumn{3}{|l|}{ Change } \\
\hline & & & & Mean (SE) & LS Mean $(95 \% \mathrm{Cl})$ & P-value \\
\hline \multirow[t]{3}{*}{$\mathrm{HbA1c}(\%)$} & Ezetimibe $(n=69)$ & $6.94(0.07)$ & $7.14(0.10)$ & $0.20(0.06)$ & $0.22(0.11,0.34)$ & $<0.001$ \\
\hline & Placebo $(n=75)$ & $6.96(0.06)$ & $7.09(0.07)$ & $0.13(0.05$ & $0.14(0.03,0.25)$ & 0.015 \\
\hline & \multicolumn{2}{|l|}{ Ezetimibe vs Placebo } & & Difference in LS mean (95\% CI) & $0.08(-0.07,0.23)$ & 0.281 \\
\hline \multirow[t]{3}{*}{ Glyocalbumin (\%) } & Ezetimibe $(n=69)$ & $17.16(0.26)$ & $17.14(0.30)$ & $-0.02(0.17)$ & $-0.02(-0.37,0.34)$ & 0.919 \\
\hline & Placebo $(n=75)$ & $17.21(0.27)$ & $17.20(0.29)$ & $-0.01(0.17)$ & $-0.02(-0.37,0.33)$ & 0.918 \\
\hline & \multicolumn{2}{|l|}{ Ezetimibe vs Placebo } & & Difference in LS mean $(95 \% \mathrm{Cl})$ & $0.00(-0.47,0.47)$ & 1.000 \\
\hline \multirow[t]{3}{*}{ Fasting plasma glucose (mg/dL) } & Ezetimibe $(n=69)$ & $124.7(2.6)$ & $128.0(2.8)$ & $3.3(3.0)$ & $6.6(1.1,12.1)$ & 0.019 \\
\hline & Placebo $(n=75)$ & $126.7(2.0)$ & $135.4(2.9)$ & $8.7(2.5)$ & $11.4(6.1,16.7)$ & $<0.001$ \\
\hline & \multicolumn{2}{|l|}{ Ezetimibe vs Placebo } & & Difference in LS mean (95\% CI) & $-4.8(-12.1,2.5)$ & 0.194 \\
\hline
\end{tabular}

CI) between treatment groups (ezetimibe vs. placebo) at 24 weeks were statistically significant except for HDL-C: LDL-C: $-21.05 \%(-25.06,-17.03) \mathrm{P}<0.001$; total cholesterol: $-13.54 \%(-16.66,-10.42) \mathrm{P}<0.001$; HDL-C: $2.45 \%$ $(-1.71,6.61) \mathrm{P}=0.25$; triglycerides: $-11.36 \%(-21.27,-1.44)$ $\mathrm{P}=0.03$; and non-HDL-C: $-19.07 \%(-22.71,-15.43) \mathrm{P}<$ 0.001 .

A summary of AEs for the ASaT population is shown in Table 3. Approximately half (49.3\%) of subjects treated with ezetimibe $10 \mathrm{mg}$ reported experiencing an $\mathrm{AE}$ and nearly $2 / 3(64.9 \%)$ of subjects receiving placebo reported experiencing an $\mathrm{AE}$ during the study period. Three subjects $(4.0 \%)$ in the ezetimibe $10 \mathrm{mg}$ group and 1 subject $(1.3 \%)$ in the placebo group reported a drugrelated AE. There were 2 subjects $(2.7 \%)$ in the ezetimibe $10 \mathrm{mg}$ group (1 cataract and 1 abdominal pain) and 5 subjects $(6.5 \%)$ in the placebo group (2 cases of

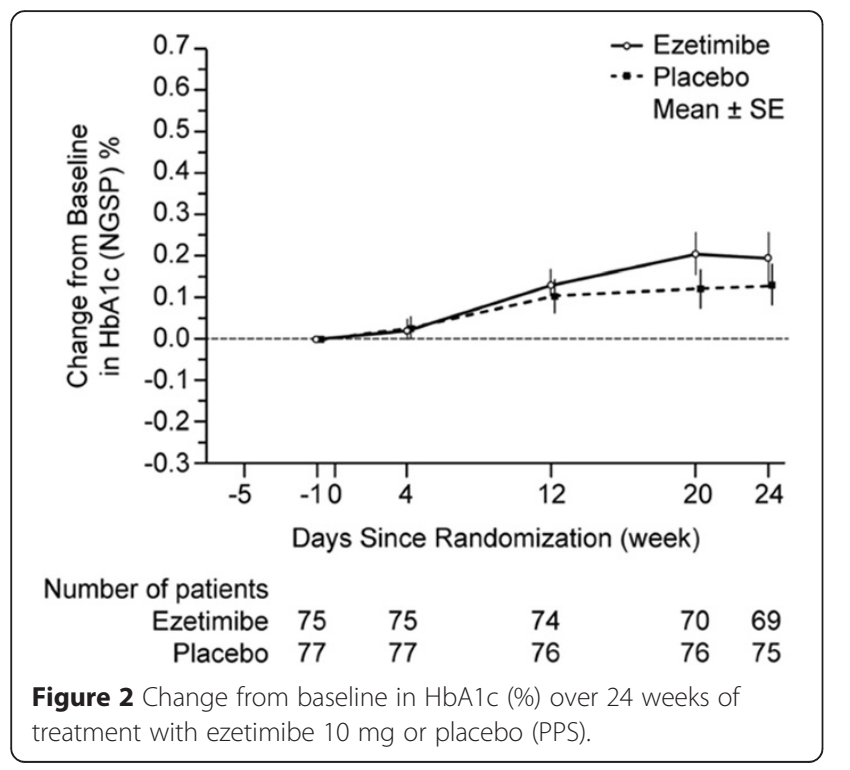

angina-one prior to study participation and one after study completion; 1 accidental overdose, 1 ankle fracture, 1 spinal osteoarthritis) that reported serious AEs. None of these AEs were considered related to study drug, nor were there any deaths in either group. The incidence of AEs of interest was generally similar between treatment groups. No serious AEs, deaths or AEs leading to study discontinuation occurred in this study.

\section{Discussion}

The results of this study showed that the ezetimibe $10 \mathrm{mg}$ group was statistically non-inferior to the placebo group with respect to change in HbA1c. Over the course of the 24 weeks of treatment, changes from baseline in glycoalbumin and fasting plasma glucose levels in both treatment groups were similar. In addition, differences in exacerbation of diabetes or changes to patients' diabetes medications due to worsening of their diabetes during the study period did not change significantly. However, differences in change from baseline lipid parameters between ezetimibe and placebo were statistically significant at 24 weeks, except for HDL-C. Ezetimibe was generally safe and well-tolerated.

Results of 2 meta-analyses suggested that treatment with statins may be associated with a small (9\%) increased risk for developing diabetes [4,19]. This was based on measures of glucose regulation such as HbA1c, and fasting plasma glucose. However, published information regarding the incident relationship of ezetimibe treatment to glucose regulations and new onset diabetes is limited. Trials that did report on glucose changes with ezetimibe treatment included subjects with diabetes or metabolic syndrome, both populations that may have impaired glucose function at study start $[20,21]$. Therefore, it is difficult to fully assess the individual impact of ezetimibe on glucose metabolism based on the published data. 


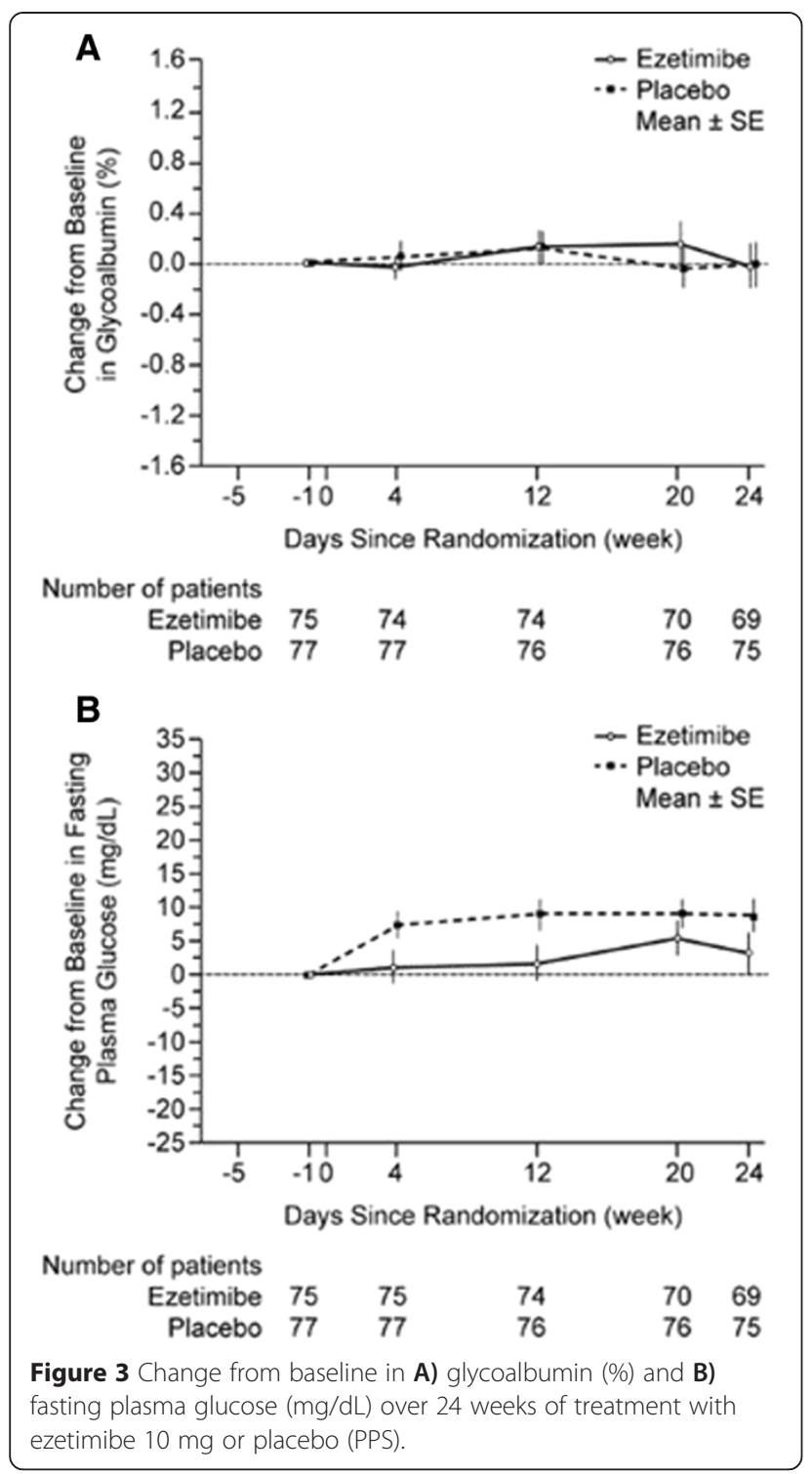

Several studies in animals have suggested that ezetimibe may have positive effects on glycemic control, including reduced weight gain, diet-induced hyperglycemia and insulin resistance [22], improvement in insulin and plasma glucose response in obese fatty rats [23], and improvement in glucose tolerance, increased insulin sensitivity, and protecting the function of beta-cells in diabetic mice [24]. In addition, one clinical trial showed that after 12 weeks of treatment with ezetimibe $10 \mathrm{mg}$ monotherapy, reductions in $\mathrm{HbA1c}$ and fasting plasma glucose were seen in Japanese patients with insulin resistance [25]. These results are consistent with those of the current trial, suggesting that ezetimibe does not confer an increased risk of glucose dysregulation. However, in the previous studies that observed neutral changes in

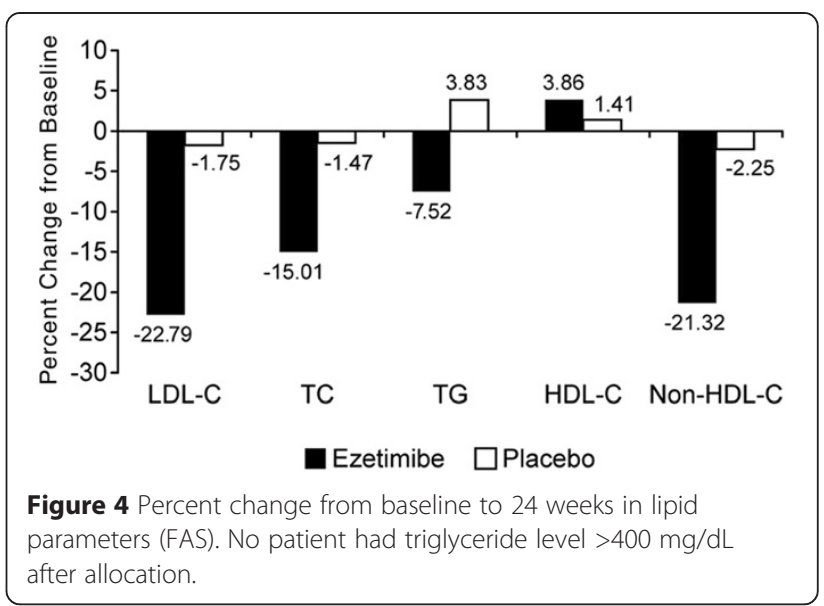

parameters of glucose metabolism and insulin sensitivity with ezetimibe monotherapy, the study populations were small, limiting the generalizability of the results to larger populations [14-16]. More recent preliminary data from two post-hoc, pooled analyses in subjects without diabetes suggest that ezetimibe monotherapy or ezetimibe added to statin does not have a negative effect on glucose metabolism $[17,18]$.

The magnitude of change from baseline in lipids, including LDL-C, total cholesterol, triglycerides and HDL-C, was consistent with those demonstrated in previous 12week trials of ezetimibe monotherapy vs. placebo [26-33]. A meta-analysis of these same trials demonstrated that ezetimibe monotherapy conferred significant reductions in LDL-C, triglyceride, and total cholesterol levels as well as a significant increase in HDL-C level compared with placebo [34]. Except for the significant HDL-C increases, the results of the meta-analysis are consistent with those of the current trial in Japanese patients.

Ezetimibe $10 \mathrm{mg}$ was generally well tolerated. Most AEs were mild in intensity with few related to treatment. There were no unexpected AEs reported based on what has been observed in other clinical trials with ezetimibe monotherapy [34].

Table 3 Summary of adverse events

\begin{tabular}{lll}
\hline & $\begin{array}{l}\text { Ezetimibe } \\
\mathbf{n ~ ( \% )}\end{array}$ & $\begin{array}{l}\text { Placebo } \\
\mathbf{n ~ ( \% )}\end{array}$ \\
\hline Subjects in population & 75 & 77 \\
With $\geq 1$ adverse events & $37(49.3)$ & $50(64.9)$ \\
With drug-related adverse events & $3(4)$ & $1(1.3)$ \\
With serious adverse events & $2(2.7)$ & $5(6.5)$ \\
Discontinued due to an adverse event & $1(1.3)$ & $1(1.3)$ \\
Discontinued due to a serious adverse event & $0(0)$ & $1(1.3)$ \\
\hline
\end{tabular}


This study had some limitations and the results must be interpreted with caution. The population was limited to Japanese patients and may not be generalizable to other populations. The short-term safety profile of ezetimibe monotherapy appears to be similar to placebo. This shortterm study limits the ability to extrapolate the results to long-term profiles. However, the recently completed IMPROVE-IT trial assessed the incremental cardiovascular benefit of LDL-C lowering over 7 years with ezetimibe $10 \mathrm{mg}$ added to simvastatin (mainly $40 \mathrm{mg}$ ) compared with simvastatin monotherapy in patients presenting with acute coronary syndromes [35-37]. The study investigators reported that the trial met its primary and secondary composite efficacy endpoints.

\section{Conclusions}

In conclusion, these data suggest that treatment with ezetimibe $10 \mathrm{mg}$ does not result in dysregulation of glucose metabolism over 24 weeks in Japanese patients with type 2 diabetes and hypercholesterolemia. Ezetimibe was generally well-tolerated with reduction of atherogenic lipid parameters.

\section{Additional file}

Additional file 1: Redacted trial protocol for study 367 entitled: Examination of the effect of ezetimibe on glucose metabolism: Randomized, double-blind, placebo-controlled study in type 2 diabetes mellitus patients with hypercholesterolemia - Phase 4.

\section{Abbreviations \\ AEs: Adverse events; ASaT: All subjects as treated; CAD: Coronary artery disease; Cl: Confidence interval; FAS: Full analysis set; HbA1c: Hemoglobin A1c; HDL-C: High-density lipoprotein cholesterol; LDL-C: Low-density lipoprotein cholesterol; LS: Least squares; PPS: Per protocol set.}

\section{Competing interests}

IS, KA, TK, and NO are employees of MSD KK and may own stock or hold stock options in the company. MEH and AMT are employees of Merck \& Co., Inc. and may own stock or hold stock options in the company.

\section{Authors' contributions}

All authors are responsible for the work described in this paper. All authors were involved in at least one of the following: [conception, design, acquisition, analysis, statistical analysis, interpretation of data] and [drafting the manuscript and/or revising/reviewing the manuscript for important intellectual content]. All authors provided final approval of the version to be published and they agree to be accountable for all aspects of the work ensuring that questions related to the accuracy or integrity of any part of the work are appropriately investigated and resolved.

\section{Acknowledgements}

Carol Zecca of Merck \& Co., Inc. provided editorial support.

\section{Funding Statement}

This study was funded by MSD KK, Tokyo, Japan.

\section{Author details}

${ }^{1}$ MSD KK, Japan development, Clinical research, Kitanomaru Square, 1-13-12 Kudan-kita, Chiyoda-ku 102-8667, Tokyo, Japan. ${ }^{2}$ Merck \& Co., Inc, Kenilworth, NJ, USA.
Received: 13 January 2015 Accepted: 16 April 2015

Published online: 01 May 2015

\section{References}

1. Assmann G, Schulte $H$. The Prospective Cardiovascular Munster (PROCAM) study: prevalence of hyperlipidemia in persons with hypertension and/or diabetes mellitus and the relationship to coronary heart disease. Am Heart J. 1988;116:1713-24.

2. Ishikawa M, Namiki A, Kubota T, Yajima S, Fukazawa M, Moroi M, et al. Effect of pravastatin and atorvastatin on glucose metabolism in nondiabetic patients with hypercholesterolemia. Intern Med. 2006;45:51-5.

3. Koh KK, Quon MJ, Han SH, Lee Y, Kim SJ, Shin EK. Atorvastatin causes insulin resistance and increases ambient glycemia in hypercholesterolemic patients. J Am Coll Cardiol. 2010;55:1209-16.

4. Ridker PM, Danielson E, Fonseca FA, Genest J, Gotto Jr AM, Kastelein JJ, et al. Rosuvastatin to prevent vascular events in men and women with elevated C-reactive protein. N Engl J Med. 2008;359:2195-207.

5. Sattar N, Preiss D, Murray HM, Welsh P, Buckley BM, de Craen AJ, et al. Statins and risk of incident diabetes: a collaborative meta-analysis of randomised statin trials. Lancet. 2010;375:735-42.

6. Zaharan NL, Williams D, Bennett K. Statins and risk of treated incident diabetes in a primary care population. Br J Clin Pharmacol. 2013;75:1118-24.

7. Lim S, Oh PC, Sakuma I, Koh KK. How to balance cardiorenometabolic benefits and risks of statins. Atherosclerosis. 2014;235:644-8.

8. Preiss D, Seshasai SR, Welsh P, Murphy SA, Ho JE, Waters DD, et al. Risk of incident diabetes with intensive-dose compared with moderate-dose statin therapy: a meta-analysis. JAMA. 2011;305:2556-64.

9. Garcia-Calvo M, Lisnock J, Bull HG, Hawes BE, Burnett DA, Braun MP, et al. The target of ezetimibe is Niemann-Pick C1-Like 1 (NPC1L1). Proc Natl Acad Sci U S A. 2005;102:8132-7.

10. Hawes BE, O'Neill KA, Yao X, Crona JH, Davis Jr HR, Graziano MP, et al. In vivo responsiveness to ezetimibe correlates with niemann-pick C1 like-1 (NPC1L1) binding affinity: Comparison of multiple species NPC1L1 orthologs. Mol Pharmacol. 2007;71:19-29.

11. Dagli N, Yavuzkir M, Karaca I. The effects of high dose pravastatin and low dose pravastatin and ezetimibe combination therapy on lipid, glucose metabolism and inflammation. Inflammation. 2007;30:230-5.

12. Tamaki N, Ueno H, Morinaga $Y$, Shiiya T, Nakazato M. Ezetimibe ameliorates atherosclerotic and inflammatory markers, atherogenic lipid profiles, insulin sensitivity, and liver dysfunction in Japanese patients with hypercholesterolemia. J Atheroscler Thromb. 2012;19:532-8.

13. Yagi S, Akaike M, Aihara K, Iwase T, Ishikawa K, Yoshida S, et al. Ezetimibe ameliorates metabolic disorders and microalbuminuria in patients with hypercholesterolemia. J Atheroscler Thromb. 2010;17:173-80.

14. Gonzalez-Ortiz M, Martinez-Abundis E, Kam-Ramos AM, Hernandez-Salazar E, Ramos-Zavala MG. Effect of ezetimibe on insulin sensitivity and lipid profile in obese and dyslipidaemic patients. Cardiovasc Drugs Ther. 2006;20:143-6.

15. Kikuchi K, Nezu U, Inazumi K, Miyazaki T, Ono K, Orime K, et al. Double-blind randomized clinical trial of the effects of ezetimibe on postprandial hyperlipidaemia and hyperglycaemia. J Atheroscler Thromb. 2012;19:1093-101.

16. Kishimoto M, Sugiyama T, Osame K, Takarabe D, Okamoto M, Noda M. Efficacy of ezetimibe as monotherapy or combination therapy in hypercholesterolemic patients with and without diabetes. J Med Invest. 2011;58:86-94

17. Toth PP, Catapano A, Farnier M, Foody J, Tomassini JE, Jensen E, et al. Changes in Fasting Glucose Levels Following Longer-term Treatment with Simvastatin Monotherapy and Combined Ezetimibe + Simvastatin [abstract]. JACC. 2014;63:A1313.

18. Toth PP, Catapano A, Farnier M, Foody J, Tomassini JE, Jensen E, et al. Effects of Ezetimibe, Ezetimibe Coadministered with Statins and Statin Therapies on Fasting Glucose Changes in Patients with Hypercholesterolemia [abstract]. J Clin Lipidol. 2013;7:277.

19. Rajpathak SN, Kumbhani DJ, Crandall J, Barzilai N, Alderman M, Ridker PM. Statin therapy and risk of developing type 2 diabetes: a meta-analysis. Diabetes Care. 2009;32:1924-9.

20. Robinson JG, Ballantyne CM, Grundy SM, Hsueh WA, Parving HH, Rosen JB, et al. Lipid-altering efficacy and safety of ezetimibe/simvastatin versus atorvastatin in patients with hypercholesterolemia and the metabolic syndrome (from the WYMET study). Am J Cardiol. 2009;103:1694-702.

21. Uemura Y, Watarai M, Ishii H, Koyasu M, Takemoto K, Yoshikawa D, et al. Atorvastatin $10 \mathrm{mg}$ plus ezetimibe $10 \mathrm{mg}$ compared with atorvastatin 
$20 \mathrm{mg}$ : impact on the lipid profile in Japanese patients with abnormal glucose tolerance and coronary artery disease. J Cardiol. 2012;59:50-6.

22. Labonte ED, Camarota LM, Rojas JC, Jandacek RJ, Gilham DE, Davies JP, et al. Reduced absorption of saturated fatty acids and resistance to diet-induced obesity and diabetes by ezetimibe-treated and Npc111-/mice. Am J Physiol Gastrointest Liver Physiol. 2008;295:G776-83.

23. Deushi M, Nomura M, Kawakami A, Haraguchi M, Ito M, Okazaki M, et al. Ezetimibe improves liver steatosis and insulin resistance in obese rat model of metabolic syndrome. FEBS Lett. 2007;581:5664-70.

24. Zhong Y, Wang J, Gu P, Shao J, Lu B, Jiang S. Effect of ezetimibe on insulin secretion in db/db diabetic mice. Exp Diabetes Res. 2012;2012:420854.

25. Tsunoda T, Nozue T, Yamada M, Mizuguchi I, Sasaki M, Michishita I. Effects of ezetimibe on atherogenic lipoproteins and glucose metabolism in patients with diabetes and glucose intolerance. Diabetes Res Clin Pract. 2013:100:46-52.

26. Ballantyne CM, Houri J, Notarbartolo A, Melani L, Lipka L, Suresh R, et al. Effect of ezetimibe coadministered with atorvastatin in 628 patients with primary hypercholesterolemia: a prospective, randomized, double-blind trial. Circulation. 2003;107:2409-15.

27. Bays HE, Ose L, Fraser N, Tribble DL, Quinto K, Reyes R, et al. A multicenter, randomized, double-blind, placebo-controlled, factorial design study to evaluate the lipid-altering efficacy and safety profile of the ezetimibe/simvastatin tablet compared with ezetimibe and simvastatin monotherapy in patients with primary hypercholesterolemia. Clin Ther. 2004;26:1758-73.

28. Davidson MH, McGarry T, Bettis R, Melani L, Lipka LJ, LeBeaut AP, et al. Ezetimibe coadministered with simvastatin in patients with primary hypercholesterolemia. J Am Coll Cardiol. 2002;40:2125-34.

29. Dujovne CA, Ettinger MP, McNeer JF, Lipka LJ, LeBeaut AP, Suresh R, et al. Efficacy and safety of a potent new selective cholesterol absorption inhibitor, ezetimibe, in patients with primary hypercholesterolemia. Am J Cardiol. 2002;90:1092-7.

30. Goldberg AC, Sapre A, Liu J, Capece R, Mitchel YB. Efficacy and safety of ezetimibe coadministered with simvastatin in patients with primary hypercholesterolemia: a randomized, double-blind, placebo-controlled trial. Mayo Clin Proc. 2004;79:620-9.

31. Kerzner B, Corbelli J, Sharp S, Lipka L, Melani L, LeBeaut A, et al. Efficacy and safety of ezetimibe coadministered with lovastatin in primary hypercholesterolemia. Am J Cardiol. 2003:91:418-24.

32. Knopp RH, Gitter H, Truitt T, Bays H, Manion CV, Lipka LJ, et al. Effects of ezetimibe, a new cholesterol absorption inhibitor, on plasma lipids in patients with primary hypercholesterolemia. Eur Heart J. 2003;24:729-41.

33. Melani L, Mills R, Hassman D, Lipetz R, Lipka L, LeBeaut A, et al. Efficacy and safety of ezetimibe coadministered with pravastatin in patients with primary hypercholesterolemia: a prospective, randomized, double-blind trial. Eur Heart J. 2003;24:717-28.

34. Pandor A, Ara RM, Tumur I, Wilkinson AJ, Paisley S, Duenas A, et al. Ezetimibe monotherapy for cholesterol lowering in 2,722 people: systematic review and meta-analysis of randomized controlled trials. J Intern Med. 2009;265:568-80.

35. Blazing MA, Giugliano RP, Cannon CP, Musliner TA, Tershakovec AM, White $J A$, et al. Evaluating cardiovascular event reduction with ezetimibe as an adjunct to simvastatin in 18,144 patients after acute coronary syndromes: final baseline characteristics of the IMPROVE-IT study population. Am Heart J. 2014;168:205-12.

36. Blazing MA, Guigliano R, Cannon CP, Musliner TA, Tershakovec AM, White $J A$, et al. IMProved Reduction of Outcomes: Vytorin Efficacy International Trial: On-treatment analysis [abstract], Presented at the American Heart Association Scientific Sessions. 2014

37. Cannon CP, On behalf of the IMPROVE-IT investigators. IMProved Reduction of Outcomes: Vytorin Efficacy International Trial [abstract], Presented at the American Heart Association Scientific Sessions. 2014.

\section{Submit your next manuscript to BioMed Central and take full advantage of:}

- Convenient online submission

- Thorough peer review

- No space constraints or color figure charges

- Immediate publication on acceptance

- Inclusion in PubMed, CAS, Scopus and Google Scholar

- Research which is freely available for redistribution 